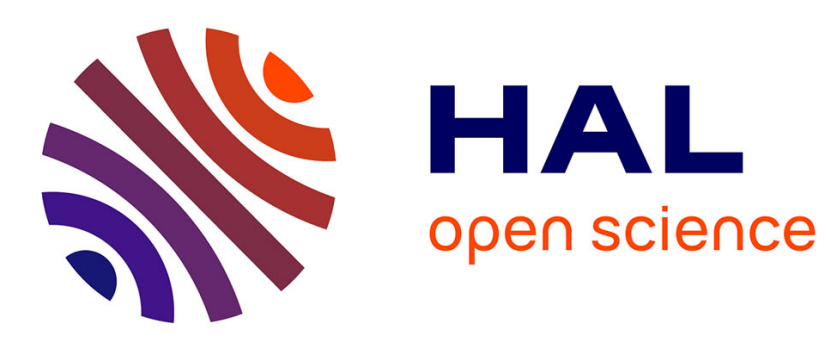

\title{
Une indication géographique pour détourner les patrimoines pastoraux? Le cas du queso cotija (mexique)
}

\author{
Thierry Linck, Esteban Barragan Lopez
}

\section{- To cite this version:}

Thierry Linck, Esteban Barragan Lopez. Une indication géographique pour détourner les patrimoines pastoraux? Le cas du queso cotija (mexique). Autrepart - Revue de sciences sociales au Sud, 2009, 2009/2 n50 (50), pp.187-202. 10.3917/autr.050.0187 . hal-02653881

\section{HAL Id: hal-02653881 \\ https://hal.inrae.fr/hal-02653881}

Submitted on 29 May 2020

HAL is a multi-disciplinary open access archive for the deposit and dissemination of scientific research documents, whether they are published or not. The documents may come from teaching and research institutions in France or abroad, or from public or private research centers.
L'archive ouverte pluridisciplinaire HAL, est destinée au dépôt et à la diffusion de documents scientifiques de niveau recherche, publiés ou non, émanant des établissements d'enseignement et de recherche français ou étrangers, des laboratoires publics ou privés. 


\section{UNE INDICATION GÉOGRAPHIQUE POUR DÉTOURNE LES PATRIMOINES PASTORAUX ? LE CAS DU QUESO COTIJA (MEXIQUE)}

Thierry Linck et Esteban Barragan

Presses de Sciences Po | Autrepart

$2009 / 2-n^{\circ} 50$

pages 187 à 202

ISSN 1278-3986

Article disponible en ligne à l'adresse:

http://www.cairn.info/revue-autrepart-2009-2-page-187.htm

Pour citer cet article :

Linck Thierry et Barragan Esteban, « Une indication géographique pour détourne les patrimoines pastoraux ? Le cas du queso Cotija (Mexique) »,

Autrepart, 2009/2 n 50, p. 187-202. DOI : 10.3917/autr.050.0187

Distribution électronique Cairn.info pour Presses de Sciences Po.

(c) Presses de Sciences Po. Tous droits réservés pour tous pays.

La reproduction ou représentation de cet article, notamment par photocopie, n'est autorisée que dans les limites des conditions générales d'utilisation du site ou, le cas échéant, des conditions générales de la licence souscrite par votre établissement. Toute autre reproduction ou représentation, en tout ou partie, sous quelque forme et de quelque manière que ce soit, est interdite sauf accord préalable et écrit de l'éditeur, en dehors des cas prévus par la législation en vigueur en France. II est précisé que son stockage dans une base de données est également interdit. 


\title{
Une indication géographique pour détourner les patrimoines pastoraux ? Le cas du queso Cotija (Mexique)
}

\author{
Thierry Linck*, Esteban Barragan**
}

\section{Introduction : les objets et les enjeux de la protection}

Les indications géographiques (IG) instaurées en 199 (d) le cadre des accords TRIPS (Trade Related aspects of Intellectual Property Klghts) sont destinées à protéger les produits dont un caractère spécifique est lié à l'origine. La protection, située sur le seul cadre des échanges internationaux, ne concerne que les dénominations, dont l'usage est placé sous le régime de la propriété intellectuelle. Le dispositif mis en place sous l'égide de l'OMC s'intéresse ainsi bien davantage à la provenance qu'à l'origine des produits : il ne pose aucune contrainte sur la nature et l'intensité de leur lien au territoire ou sur les systèmes productifs d'où ils sont issus. En toute logique, ces responsabilités incombent aux garants de la propriété intellectuelle, c'est-à-dire aux États signataires de l'accord international. C'est à eux de décider s'il convient, dans le cadre des politiques publiques qu'ils mettent en œuvre et des objectifs qu'ils se donnent, de construire les règles destinées à garantir l'origine du produit au-delà d'une simple référence à un lieu et, le cas échéant, d'arbitrer les tensions entre producteurs, opérateurs et usagers. Tous les États signataires de l'accord international n'assument pas cette responsabilité au même degré ni dans le même sens. Ainsi, si les pays anglo-saxons sont généralement partisans d'une reconnaissance sui generis des indications géographiques, l'Union européenne prône des dispositifs fondés sur un cahier des charges contraignant, mais dont l'intérêt et la portée en termes de protection de l'environnement et des patrimoines locaux font débat. [Marie-Vivien, Thévenod-Mottet, 2007]. Nous verrons que le Mexique s'en tient à une interprétation littérale.

Il reste que l'octroi d'une IG assure une certaine reconnaissance du produit, en renforce la réputation et induit un élargissement et une segmentation des

\footnotetext{
* Économiste, INRA-SAD, Corte, Corse, linck@corte.inra.fr

** Géographe, El Colegio de Michoacan, Zamora, Michoacán, México, estebar@colmich.edu.mx
} 
marchés qui suscitent de nouvelles attentes et, convoitises et portent des dynamiques qui ne sont pas sans effet sur les produits et les systèmes productifs. Les promesses d'accès à de nouveaux débouchés, urbains ou extérieurs, annoncent des changements d'échelle, éveillent l'intérêt de nouveaux opérateurs, suscitent des innovations techniques qui touchent potentiellement l'ensemble de la chaîne productive, favorisent l'émergence de nouvelles modalités de commercialisation et d'organisation et induisent un risque d'altération des matières premières, des procédés et, au final, des produits eux-mêmes.

L'octroi d'une IG suscite ainsi le développement de nouveaux modes d'interaction. À un univers largement structuré autour de savoirs informels, techniques et relationnels se substitue un ensemble de règles et de codes formels qui touchent la caractérisation du produit, l'élaboration de règlements techniques et impose un respect plus strict des règles, notamment sanitaires, qui cadrent l'ensemble de l'activité. Aussi précis qu'ils soient, les dispositifs institutionnels qui portent le passage de conventions domestiques à des conventions de type industriel, sont par nature incomplets. Et, dans la mesure où ils sont l'expression d'une volonté politique par essence contingente, ils ne sont jamais parfaitement neutres. La notoriété associée à l'origine peut ainsi être mise au service d'objectifs spécifiques (de politiques sectorielles ou d'aménagement, par exemple) ou encore détournée au bénéfice d'intérêts particuliers, aux dépens, parfois, du produit et des systèmes productifs originels. Dans ce sens, au-delà des risques d'imitation et de contrefaçon sur les marchés extérieurs, la mise en place des IG appelle une réflexion plus large sur la problématique de la protection et un examen attentif de l'intérêt et des inconvénients que les IG présentent pour les territoires et les nations concernées.

Nous avons choisi de traiter cette question en considérant que la spécificité d'un produit d'origine tient à l'incorporation de ressources non transférables propres aux territoires et aux sociétés qui en assurent la production. Cette posture ouvre des perspectives intéressantes du fait que les ressources incorporées sont dans une très large mesure dotées d'attributs de biens patrimoniaux [Linck, 2007]. En effet, les biens environnementaux (dans la mesure où ils sont le fruits d'actions d'aménagement parfois très anciennes), les savoirs techniques et organisationnels (ainsi que les systèmes cognitifs qui les structurent), peuvent être considérés à la fois comme les résultats d'actions collectives inscrites dans des temporalités longues et comme des biens non marchands. Leur incorporation dans le processus productif est pourtant à l'origine d'une plus-value (« le prix de la qualité ») qui découle de la mobilisation d'une exclusivité d'usage. Considérée sous cet angle, la problématique de la protection peut être abordée en lien avec une réflexion sur le partage de la valeur, les conflits d'usage et, en définitive, sur les dispositifs d'appropriation patrimoniale [Linck, 2005]. Elle peut enfin être conduite sur une base plus objective dans la mesure où l'objet de la protection n'est pas la réputation (considérée pour ellemême) mais bien la ressource patrimoniale (dont elle découle).

Dans cette perspective, la qualification d'un produit peut être considérée comme une construction sociale : elle repose sur la mise en œuvre de choix 
collectifs portant sur la construction de règles destinée à cadrer en dernier ressort l'identification, l'appropriation, l'activation et la gestion de ressources territoriales. Dans la mesure où ils sont instruits par des projets et des critères spécifiques, portés par des rapports de force marqués par leur cadre institutionnels, ces choix collectifs sont par nature contingents.

Ces questions prennent un sens particulier au Mexique et dans de nombreux pays d'Amérique latine où le relief, l'absence de voies de communication et de ressources hydriques marquent les limites de la diffusion du modèle productiviste. Ces espaces marginalisés se prêtent mal à des systèmes techniques largement fondés sur l'usage de moyens de production d'origine industrielle, d'énergies fossiles et étroitement associés au grand négoce de l'alimentation. De fait et dans une certaine mesure, l'isolement a des vertus protectrices. Ainsi, dans les montagnes de l'Occident mexicain, le désintérêt des agences publiques de développement et autres promoteurs de l'agriculture «moderne » a souvent permis le maintien d'une économie informelle qui puise sa force dans l'intensité de son ancrage territorial. Dans ces territoires marginalisés, les productions fromagères peuvent constituer un excellent indicateur de cet ancrage et de cette capacité à résister. Le fromage traditionnel constitue en effet une expression de systèmes productifs complexes où les cultures, les élevages, les activités de transformation ainsi que les usages de consommation entretiennent des liens intenses, fondés sur une logique de valorisation globale des ressources locales et que structurent des trames cognitives spécifiques. Mais les vertus protectrices de l'isolement ont leurs limites. Les aspirations de la population locale (en matière, notamment, de santé et d'éducation) ajoutent leurs effets à la concurrence des agricultures de plaine et des industries alimentaires qui prive les régions isolées de débouchés tant pour ce qui concerne les productions animales que végétales. La déprise agricole et la pauvreté alimentent un exode rural souvent massif accélérant ainsi le délitement du lien social local. Les vertus protectrices de l'isolement sont aujourd'hui d'autant plus limitées que les attentes des consommateurs urbains [Fischler, 2001 ; Poulain, 2005] poussent les industries de l'alimentation à diversifier leur offre en mobilisant des valeurs symboliques censées garantir « l'authenticité » de leurs produits. Autrefois oubliés, ces espaces marginalisés deviennent ainsi - grâce aux dispositifs de qualification - parfois l'objet de nouvelles convoitises et de nouvelles menaces : l'appropriation d'un nom et des représentations qui lui sont associées. Cette inflexion ne remet en cause ni les orientations marchandes ni les choix techniques dominants à l'échelle nationale : loin de s'inscrire dans un projet de développement territorial ou dans une logique d'intensification écologique, elle ne correspond qu'à une nouvelle étape du processus d'accumulation primitive.

L'exemple du queso Cotija est emblématique de la place qu'occupent au Mexique les démarches de qualification sous indication géographique. D'une part, parce que le développement du produit s'inscrit dans une scène où apparaissent clairement l'emprise du contexte institutionnel, le poids et les rigidités de l'encadrement technique ainsi que l'enfermement de la démarche lancée par les producteurs dans un jeu où dominent sans conteste les options doctrinales de l'État et 
les intérêts du complexe agroalimentaire. D'autre part, parce que l'entêtement des producteurs à défendre l'ancrage territorial de leur produit, leur engagement autour d'un projet de développement territorial, leurs premiers succès et les avatars qui ont suivi font désormais l'objet d'un débat national dont les enjeux dépassent de loin le seul cadre des Sierras de Jalmich.

Cette question est également placée au cœur de notre argumentaire. Nous le construirons en ouvrant une réflexion sur les enjeux liés à la définition du produit. Cette première étape nous conduira à mette en évidence l'importance du lien entre produit et système productif, ce qui nous permettra de mieux apprécier les enjeux liés à la protection du queso Cotija et le caractère asymétrique des rapports avec les filières laitières. L'appropriation dans la mesure où elle met en scène les rapports entre le produit et la ressource territoriale implique une capacité à gérer l'un et l'autre : elle doit également être située en fonction d'un objectif. Le projet défendu par le collectif des producteurs s'inscrit résolument dans une démarche d'ancrage et de développement territoriaux, comme en témoigne, entre autres éléments, la délimitation de l'aire de dénomination ou les prescriptions en matière de race animale. À l'opposé, le projet porté par l'État, tout comme le cadre institutionnel de son action, relève pleinement d'une perspective sectorielle et de diffusion des recettes du productivisme. Nous verrons ainsi que le blocage de la démarche de qualification tient aux options techniques définies par l'État, mais également aux contraintes sanitaires et aux procédures qu'il met en œuvre. En conclusion, les derniers développements d'un processus encore inachevé n'incitent pas à l'optimisme : la nature des aides publiques octroyées et le compromis obtenu avec la création d'une marque collective n'impliquant aucune obligation de contrôle (et donc de respect) du cahier des charges suggèrent que le queso Cotija est en passe de ne plus appartenir aux éleveurs des Altos de Jalmich et que faute d'avoir pu maintenir ses liens avec son territoire d'origine il risque fort de cesser d'exister.

\section{Le queso Cotija : I'histoire, l'espace, la société}

Nous aurions tort de définir le queso Cotija comme une simple pâte pressée, une tomme similaire au Cantal par son aspect et ses dimensions. Le queso Cotija est avant tout un fromage pastoral issu d'élevages à vocation viande dont les dimensions et les modalités d'affinage permettent la conservation et le transport à dos de mulet sur des chemins incertains et des distances considérables. Il ne s'agit pas d'une simple question de nuance : la première définition repose sur une approche taxonomique qui réduit le fromage à son seul statut de marchandise, la seconde met en avant le lien qui le rattache au système productif d'où il est issu et qui lui confère son authenticité et l'essentiel de ses caractéristiques sensorielles. Celles-ci tiennent pour une large part aux savoirs mobilisés dans les activités d'élevage et de transformation ainsi qu'à à la richesse des flores pâturées et des flores lactiques. Il est bien question là de deux perspectives antinomiques dans la 
mesure où elles instruisent des approches opposées de la protection et de l'appropriation d'un produit d'origine ${ }^{1}$.

Considéré dans cette perspective, le queso Cotija apparaît comme un produit profondément ancré dans l'histoire du Mexique. Il est emblématique de ces espaces qui échappaient (ou échappent encore) à l'emprise directe de la puissance publique coloniale puis nationale et dont la conquête et la colonisation ont été assurées par une population métisse ou d'origine espagnole : les rancheros [Barragan et Linck, 1993 ; Lloyd, 1988 ; Katz, 1991].

Dans ces territoires isolés et délaissés, la violence, et surtout, le cheval et la vache restent, de nos jours encore, les principaux outils du contrôle de l'espace. L'élevage bovin appelle la mise en place de clôtures et conduit à l'appropriation des terres que les populations indiennes vouaient à un usage collectif (Linck, 2001). Si le cheval permet de développer une économie pastorale sur une vaste échelle, il marque aussi la domination symbolique du ranchero, "l'homme à cheval » [Chevalier, 2006], sur l'indien, piéton, « païen » et cultivateur. L'alcool, la corruption des élites locales et la violence ont fait le reste : à quelques exceptions près (dont celle notable du Chiapas), les populations indiennes ont été éliminées des espaces rancheros. L'isolement appelle la mise en place d'une économie semi autarcique. Avant le développement, relativement récent, du réseau routier national, les sociétés rancheras étaient largement autosuffisantes. La culture du maïs, en association avec celle de courges ou de haricots, l'entretien de vergers et de potagers familiaux, les volailles, les porcs, le fromage que complétaient la chasse, la cueillette et des activités artisanales variées permettaient de satisfaire les besoins essentiels des populations locales [Barragan, 1990].

Mais l'isolement est toujours relatif : les sociétés rancheras constituent aussi des relais, des postes avancés de la société nationale. L'essor des activités commerciales a ainsi permis la fondation de centres urbains à la fin du XIX siècle : c'est notamment le cas de Cotija, sur les franges occidentales de l'axe néovolcanique, à la jointure des États du Michoacán et du Jalisco. Cette ville modeste s'est spécialisée dans le commerce à grande distance et l'expédition sur l'ensemble du territoire national du fromage auquel elle a donné son nom sans jamais en avoir vraiment produit [Moreno, 1980 ; Gonzalez et Ortiz, 1980].

Le métayage - ou, plus largement, le travail a destajo ${ }^{2}$ - est le rapport de production fondamental des sociétés rancheras. Il fixe les rapports entre maïs et élevage, il structure la division technique et sociale du travail, il règle les modalités de partage des richesses et il permet, enfin, d'expliquer la stratification et les dispositifs de régulation de la société ranchera. Le métayage repose sur une

1. Voir en particulier Poméon [2007], Boucher [2008] et Rodriguez Gomez [2002] dont les analyses sont fondées sur une définition du queso Cotija du premier type et dont les conclusions sont sensiblement éloignées des nôtres.

2. Travail à façon, utilisé également dans des activités non agricoles. Il s'agit d'un dispositif destiné à cadrer l'organisation du travail, l'accès aux ressources productives (la terre dans le cas du métayage) et organisationnelles et à valider les compétences individuelles. 
relation de confiance partagée qui donne aux sociétés rancheras une grande souplesse : le mediero assiste son patron dans la plupart de ses activités, leurs familles souvent proches par le sang ou les alliances matrimoniales, partagent fréquemment les mêmes conditions d'existence, les mêmes valeurs, les mêmes soucis et, dans une large mesure, les mêmes attentes. Il n'est pas rare qu'un métayer dont les compétences et la loyauté sont reconnues assume au sein du rancho des responsabilités de plus en plus grandes pour finalement s'en voir confier la gestion avant de se convertir lui-même en éleveur - propriétaire. La mobilité sociale, au même titre que la mobilité géographique peut ainsi être reconnue comme un trait distinctif des sociétés rancheras [Barragan, 1997 ; Linck, 2001 ; Shadow, 1990].

\section{Le système-produit}

Il est difficile de comprendre l'identité du queso Cotija sans s'aventurer au cœur des régions où il est encore produit. Ces paysages de montagne contrastent fortement avec les plaines que ces régions dominent parfois. Le relief est si accidenté qu'il ne laisse que fort peu de place pour la pratique de cultures sédentaires [Cochet, 1991]. Le sentiment d'isolement et de solitude est renforcé par l'absence de villages, d'habitants et de voies de communication visibles.

Les deux composants fondamentaux de l'organisation productive sont l'élevage bovin (pour la production de taurillons) et la culture itinérante du maïs. En apparence, le maïs constitue l'élément clé du système : cultivé en défriche-brûlis, c'est bien lui qui laisse les traces les plus évidentes sur le paysage. Cependant, l'essartage, le brûlage et l'abandon des parcelles après une seule campagne de culture confirment la vocation première des régions rancheras : le pastoralisme ${ }^{3}$. Les modes d'organisation et d'utilisation de l'espace répondent ainsi bien davantage aux besoins de l'élevage qu'aux exigences des cultures. La durée des cycles agricoles est pensée en fonction d'une valorisation optimale du potentiel fourrager : la fin de la jachère est décidée avant que le développement de la végétation pérenne ne gêne véritablement celui des graminées.

Aux deux composants qui ont été évoqués - l'élevage extensif qui donne sens au système et la culture itinérante du maiis qui structure son rapport à l'espace il convient ainsi d'en ajouter un troisième : la production de fromage. Il est produit durant la saison des pluies, entre juin et octobre, à un moment où l'abondance de fourrage permet à l'homme de prélever une part de la ration normalement destinée aux veaux : deux à trois litres tout au plus. L'éleveur, ses métayers et leurs familles rejoignent la résidence d'été - le rancho de ordeña - où les vaches allaitantes - de l'ordre d'une cinquantaine - sont regroupées avec leur veau. Réalisée à l'aube, la traite quotidienne (la ordeña) permet d'obtenir une centaine de litres de lait qui sera transformée le jour même : une fois salé, le caillé du jour est alors mélangé

3. À la différence des systèmes extensifs et productivistes, nous caractérisons le pastoralisme par un usage intensif de capital humain, un usage global des ressources locales de préférence aux intrants et équipements achetés et une internalisation des risques. 
avec celui de la veille, disposé dans son moule et pressé pour obtenir une meule d'une trentaine de kilos destinée à être affinée durant 3 ou 4 mois. Compte tenu de l'absence fréquente d'eau potable et d'électricité, la fabrication doit impérativement être réalisée sur place, avec soin et sans retard. Compte tenu de l'isolement (qui interdit virtuellement l'organisation de collectes) et de conditions d'hygiène précaires, le queso Cotija ne peut être qu'un fromage fermier au lait cru, produit sur la base d'une gestion empirique des flores microbiennes.

La production de fromage constitue un puissant régulateur. Elle favorise un usage global des ressources disponibles: ressources fourragères, excédents de maiis et forces de travail. Le queso Cotija est essentiel pour le maintien d'un équilibre durable du régime alimentaire; il apporte aussi des compléments de revenus qui ne sont en rien négligeables - de l'ordre du tiers ou davantage des recettes obtenues de la vente des taurillons - tant pour les éleveurs que pour les métayers. C'est un produit doté d'une valeur symbolique élevée : on en est fier, on l'exhibe, on le partage, on l'offre aux visiteurs de marque...

\section{Le coût de la notoriété : concurrence et contrefaçons}

Le commerce lointain depuis la ville de Cotija et, dans une certaine mesure l'ancrage territorial expliquent la grande notoriété du fromage de Cotija, sa présence dans un grand nombre de préparations culinaires sur l'ensemble du territoire la confirme. Le queso Cotija est consommé en apéritif, râpé (ou plutôt émietté), il accompagne un très grand nombre de plats traditionnels. Le terme de notoriété doit être entendu ici stricto sensu : le fromage de Cotija est connu de tous. Mais il est aussi partout reconnu comme un produit rustique, il est d'ailleurs généralement vendu à la coupe, en très petites quantités (forme adaptée aux usages de consommateurs à faibles revenus). Au Mexique, cet attribut a encore une connotation péjorative : le queso Cotija est placé au rang des produits populaires dont les qualités sensorielles sont posées comme négligeables. Cette caractéristique présente un attrait particulier qui ouvre à la grande et à la petite industrie fromagère un débouché pour les rebuts de fabrication et des produits élaborés à partir de poudre de lait ou de lactosérum, voire même de soja. Le queso Cotija est donc un produit largement imité, contrefait et altéré : la quinzaine de tonnes produites actuellement par une cinquantaine d'éleveurs dans les Altos de Jalmich ne représente aujourd'hui qu'une infime partie de la production identifiée par les mentions Cotija ou «type Cotija ». La production authentique est noyée dans une dynamique qui tire les prix vers le bas et ferme les débouchés traditionnels : en l'espace de trois décennies, la production a chuté des deux tiers et le nombre de producteurs de moitié [Barragan et al., à paraître].

\section{Une action collective construite autour d'un projet territorial}

Dans le Potrero de Herrera, au cœur des Altos de Jalmich, les capacités organisationnelles ont pris forme dans les années soixante-dix et quatre-vingts à propos 
de l'ouverture de pistes carrossables. Le développement rapide du réseau routier national, le besoin de plus en plus pressant d'accéder aux services publics de base (santé et éducation notamment), l'essor des débouchés urbains et l'épargne locale (le fonds de réserve que permet de constituer l'élevage extensif) mobilisée pour l'achat d'un véhicule ou la réalisation des travaux de terrassement ont favorisé les ententes entre voisins [Barragan et Linck, 1993]. Ces organisations se sont structurées autour d'un objectif de développement territorial et patrimonial avec, notamment l'organisation de foires régionales du fromage à Cotija, la création de Unidades de Manejo y Conservacion de la vida silvestre UMA) et, surtout, de l'association Prosierra de Jalmich à l'origine de ces initiatives et qui est censée en assurer l'orientation et la coordination. L'action militante, la signature de conventions avec les États du Michoácan et du Jalisco, le soutien des autorités municipales ont contribué à la consolidation du mouvement jusqu'au milieu de la décennie 2000, [Barragan 2005]. Simultanément, les éleveurs, confrontés aux difficultés de commercialisation du fromage, n'ont pas tardé à prendre conscience du fait que le devenir de leur région et celui de leurs propres conditions d'existence était largement lié à leur engagement dans une action commune : celle-ci prendra véritablement forme à partir de 1998 avec la création d'une Sociedad de Produccion Rural (SPR), première étape en vue de l'obtention d'une denominacion de origen $^{4}$.

La quête d'une reconnaissance officielle du queso Cotija conduite par Prosierra s'inscrit ainsi dans un projet territorial et patrimonial plus large. Il est beaucoup question de rompre l'isolement des rancherias, mais aussi d'assurer la promotion de ses produits et la pérennité des activités. La première Feria del queso est organisée en 2000. D'autres suivront et connaîtront le même succès : le fromage de Cotija s'y vend à un prix sensiblement supérieur et y trouve une reconnaissance en tant que produit de qualité. Par ce moyen, sa connotation rustique, trop souvent encore pénalisante, a pu être convertie en un argument valorisant. D'un point de vue strictement économique, le gain immédiat est limité dans la mesure où les ferias ne n'offrent pas une alternative durable aux réseaux traditionnels de commercialisation et moins encore à la concurrence des produits contrefaits. En revanche elles ont fortement contribué à améliorer l'image du produit, à réveiller la fierté des producteurs ainsi que leur optimisme. Elles ont donné sens à un mouvement collectif qui a pris corps progressivement dans la mise en place d'ateliers de dégustation et d'échanges techniques, dans la participation à des manifestations de plus grande envergure, nationales et internationales (le queso Cotija est reconnu meilleur fromage étranger de montagne à la foire internationale des fromages de Crémone en $2006^{5}$ ) ainsi que dans la recherche d'appuis institutionnels, notamment auprès du CIATEJ $^{6}$ et du Colegio de Michoacán.

4. Dispositif de qualification territoriale placé sous l'autorité de l'institut mexicain de la propriété industrielle. Ley de propiedad intelectual, art. 6. www.sice.oas.org/int_prop/nat_leg/Mexico/

5. La Jornada, Mexico, 14 novembre 2006.

6. Centro de Investigación y Asistencia Tecnológica del Estado de Jalisco, Guadalajara. 
Il faut protéger le fromage de Cotija. C'est une évidence pour les éleveurs des Altos de Jalmich qui subissent durement la concurrence des produits contrefaits et qui vivent la non reconnaissance de leur produit comme une atteinte à leur identité collective. Dans ces contrées isolées marquées par de très faibles densités de population (de l'ordre de 5 habitants par $\mathrm{km}^{2}$ ), une grande dispersion de l'habitat (les densités de localités sont parmi les plus élevées du Mexique ${ }^{7}$ ) et de grandes difficultés de communication, la mise en œuvre de ce projet exige une action collective d'autant plus difficile à mettre en œuvre que les systèmes de valeurs rancheros favorisent a priori les comportements individualistes. Il faut donc croire que l'importance des enjeux n'a pas échappé à la population locale.

Les démarches engagées en vue de l'obtention d'une dénomination d'origine sont donc étayées par un engagement collectif conséquent. Cet engagement a pris sens dans la définition d'un projet territorial structuré par une quête de reconnaissance officielle : défendre le produit, non pas simplement pour lui-même et moins encore du seul point de vue de sa valorisation marchande, mais bien plutôt pour la préservation du système productif, du mode de vie et des valeurs patrimoniales qui leur sont intimement associés. Les éleveurs ont su discerner les marqueurs de typicité de leur fromage et ce qui en détermine en dernier ressort l'authenticité : une saveur et un aspects particuliers, mais aussi et surtout un ancrage territorial qui lui est propre. Ils ont surtout clairement pressenti la nature des menaces qui pèsent sur leur fromage : les risques de banalisation et d'altération qu'entraînerait la mise en œuvre de choix techniques difficilement compatibles avec les orientations de leur système productif. Le règlement technique présenté avec le dossier de la dénomination d'origine pose des exigences précises qui constituent autant de garde-fous contre les risques d'altération, de contrefaçon et de banalisation du produit : l'aire d'appellation correspond au cœur du massif des Sierras de Jalmich où prédominent les paysages typiquement rancheros. Son découpage suit scrupuleusement les contours des versants de telle sorte que l'absence d'irrigation et la topographie interdisent le développement de cultures fourragères mécanisées ou irriguées, fermant par là les options d'intensification fondée sur une spécialisation laitière des élevages. Dans le même sens, la proscription des races laitières préserve une caractéristique essentielle du fromage de Cotija : le pastoralisme. Enfin, le même règlement technique fixe les caractéristiques du produit (taille, aspect, utilisation exclusive de laits crus et affinage) qui limitent étroitement les possibilités de production à grande échelle ${ }^{8}$. Il s'agit là de prescriptions et de restrictions d'ordre technique, les seules à pouvoir durablement assurer la protection du produit et sa pérennisation. Comme nous allons le voir, il s'agit également de mesures qui s'inscrivent à contre-courant des politiques publiques et des choix techniques qui les structurent.

Le projet gouvernemental est en effet d'une tout autre nature. Son objet n'est pas la protection de savoir-faire ou de ressources territoriales, ni même d'un

7. Censo General de Poblacion y Vivienda, México 2004.

8. Reglas de uso de la marca colectiva queso "Cotija región de origen». 
produit typique, mais simplement la mise en réserve d'un nom et de la réputation qui lui est associée et sa valorisation dans la conquête de nouveaux débouchés. L'objectif vise ainsi bien davantage le développement du produit et sa valorisation industrielle. Le fait que le cadrage institutionnel de la certification soit confié à l'Institut mexicain de la propriété industrielle (IMPI) qui relève lui-même du ministère de l'économie en témoigne, tout comme en témoigne le peu de soin généralement accordé à la définition des caractéristiques du produit protégé comme des aires d'appellation et l'absence de référence aux systèmes productifs locaux : les cas du tequila ${ }^{9}$, du café du Chiapas ${ }^{10}$, de la mangue d'Atulfo ${ }^{11}$, de la Charanda ${ }^{12}$, du dossier mezcal ${ }^{13}$ et de bien d'autres produits sont particulièrement éloquents.

\section{Un fromage fermier au lait cru ? Impossible !}

Nous l'avons souligné, le queso Cotija ne peut être que fermier. En premier lieu parce que la dispersion des unités de production et l'absence de voies de communication sûres interdisent l'organisation de la collecte. En second lieu parce que l'absence fréquente d'eau potable et d'électricité imposent un caillage et un salage rapides. Enfin, parce que le queso Cotija est un fromage pastoral : ses attributs sensoriels portent la marque d'une conduite des animaux sur parcours et d'une transformation à la ferme. Pourtant, le queso Cotija ne peut pas être reconnu comme tel : le droit mexicain ignore la catégorie «produit fermier». Mais il y a plus grave : le queso Cotija n'est pas non plus un fromage, à tout le moins il n'est pas reconnu comme tel puisque les normes sanitaires mexicaines (renforcée dans ce domaine par la signature de L'Accord de Libre Echange Nord-américain) ne reconnaissent la qualité de fromage qu'aux produits élaborés à partir de laits pasteurisés. Pourtant, nous l'avons également souligné, le queso Cotija ne peut être élaboré qu'à partir de lait cru. La préservation des flores lactiques est essentielle tant pour la construction de sa valeur sensorielle que pour la gestion des risques sanitaires. L'absence de froid artificiel et d'eau potable ne permet pas de respecter l'hygiène stricte qu'impose la pratique de la pasteurisation; le traitement rapide du lait, le salage et l'entretien de biofilms sur les ustensiles avec lesquels le lait ou le caillé sont en contact sont à la base d'une gestion spécifique des risques sanitaires davantage fondé sur une logique d'internalisation plutôt que d'évitement.

9. Les aires de production de la matière première - l'agave bleu - peuvent être désormais très éloignées du berceau originel situé au centre de l'État de Jalisco.

10. L'État du Chiapas constitue, du point de vue de la production de café, une mosaïque particulièrement complexe (altitude, exposition, pluviométrie, ensoleillement, température, mais aussi ethnique).

11. Il s'agit d'une simple variété, dont la production n'est associée à aucune contrainte spécifique.

12. Il s'agit d'une eau de vie de canne à sucre très ordinaire.

13. En cours d'instruction. Le mezcal est en fait un produit générique issu de variétés d'agaves non spécifiées et produit selon des techniques très variables selon les régions... 


\section{Une marque pour privatiser une ressource collective}

Cette double contrainte laissait peu de chances de succès aux démarches engagées par le collectif de producteurs. En fait, le refus opposé par l'IMPI repose sur un argument d'une toute autre nature : à force d'imitations et de contrefaçons, le queso Cotija est désormais officiellement identifié comme un produit générique : la mention de l'origine ne peut dès lors faire l'objet d'aucune protection. Il est vrai que la production de fromage authentique ne représente qu'une part infime des fromages vendus sous l'appellation Cotija et pour la plupart contrefaits et dénaturés...

Si le queso Cotija ne peut prétendre à la protection qu'est censée apporter la Denominacion de origen, les démarches engagées et la notoriété que lui a valu son succès à la foire de Crémone ont en quelque sorte « révélé » son existence aux yeux des pouvoirs publics, mettant à jour un nouvel écueil : la foi naïve dans les vertus du progrès technique que partagent la plupart des agents de l'administration et bon nombre d'agronomes mexicains [Linck, 1988]. L'initiative prise par le gouvernement de l'État du Jalisco est à cet égard particulièrement significative : une unité de pasteurisation sera livrée clé en main sur le territoire du municipe de Santa Maria del Oro, sur les franges occidentales de l'aire de dénomination. D'un point de vue technique, l'option réduit à néant les efforts engagés par le collectif dans la construction du cahier des charges: les fromages que l'unité produira perdront leur caractère fermier, seront issus de laits pasteurisés et, à terme, d'élevages intensifs situés hors de l'aire de dénomination. D'un point de vue organisationnel, l'initiative conduira inévitablement à briser l'unité du collectif : compte tenu des difficultés de communication, la collecte ne pourra pas couvrir la totalité de l'aire de dénomination, ce qui entraînera l'exclusion de fait des producteurs du Michoacán. Du point de vue de la cohérence du processus de qualification, l'imposition, dans une démarche descendante, de ces choix techniques et organisationnels peut être interprétée comme une action de dépossession : elle équivaut à une expropriation des savoirs techniques et organisationnels locaux ainsi que des capacités d'accès aux ressources environnementales. En outre, ces choix ne sont pas sans effet sur le produit lui-même : l'intensification des élevages et l'appauvrissement des flores lactiques entraîneront une perte majeure de typicité. Le nouveau queso Cotija jouira un temps de la notoriété que lui aura valu la lutte des producteurs, mais il perdra irrémédiablement sa spécificité et tout impact véritable en terme de développement territorial et de maintien du pastoralisme.

Si la dépossession des savoirs locaux, des fruits de l'action collective et du produit lui-même passe par l'imposition de recettes techniques, elle trouve également un soutien appréciable dans les dispositifs institutionnels qui cadrent l'activité économique. La dénomination d'origine a été refusée, mais la Sociedad de Produccion Rural a obtenu (en novembre 2005) le bénéfice d'une marque collective : «queso Cotija region de origen ». Cette mesure compensatoire présentée comme une étape préalable à l'octroi éventuel de la denominacion de origen a pu 
être perçue comme un succès : la décision couronne les efforts du collectif, et assure une reconnaissance officielle à la fois du queso Cotija, de l'engagement collectif et du règlement technique élaboré pour le dossier de la dénomination. Mais il reste que cette reconnaissance est purement formelle : le règlement technique (donc les contours de l'aire de dénomination, l'origine des laits et les procédés de fabrication) ne fait l'objet d'aucune obligation de contrôle et donc d'aucun engagement véritable ${ }^{14}$. Dans ce sens, l'octroi de la marque collective, loin de faire obstacle au projet de laiterie lancé par le gouvernement de l'État du Jalisco l'a plutôt encouragé. Dans son principe même, elle s'inscrit dans une démarche de marchandisation et de privatisation de ressources collectives. Comme pour toute marque commerciale, le droit de propriété qui lui est associé doit être entendu dans son sens le plus absolu. Son détenteur bénéficie à la fois d'une exclusivité d'usage et du droit de gérer librement la marque, sans autres contraintes ou contrôles que ceux que son détenteur juge de sa convenance. La marque collective déposée, le projet de laiterie lancé, rien ne s'opposait à la mise en œuvre de la dernière étape du processus de dépossession : en mai 2008, les producteurs du Jalisco prennent le contrôle de la Sociedad de Produccion Rural, se désolidarisent de l'association Prosierra et ne tardent pas à engager des négociations avec une firme nord-américaine qui propose de commercialiser en exclusivité le nouveau queso Cotija. Elle culminera lorsqu'il sera établi - ce qui ne saurait tarder que le produit est en conformité avec les normes mexicaines et nord-américaines ${ }^{15}$.

\section{Conclusion : à qui appartient le queso Cotija?}

Le queso Cotija, du moins celui qui est encore produit dans les replis les plus isolés de la Sierra de Jalmich est au plein sens du terme une ressource patrimoniale. D'une part, parce que ce fromage fait corps avec le système pastoral qui structure la société ranchera, tant pour ce qui concerne la conduite des animaux, l'entretien du potentiel fourrager que les mode de gestion des flores microbiennes mobilisées dans la fabrication du produit. D'autre part, parce qu'il s'agit d'une ressource collective portée par des dispositifs cognitifs (savoirs techniques et relationnels) qui structurent la société locale et ont été construits collectivement dans les temporalités longues du lien intergénérationnel. Enfin, parce que le queso Cotija est un puissant marqueur identitaire, générateur de lien social tant dans la sphère des activités productives que dans celle de l'entretien de la sociabilité.

Considéré en tant que bien patrimonial, il ne fait aucun doute que le queso Cotija est un bien collectif de la société ranchera considérée dans sa globalité : à ce titre, il appartient à tous ses membres selon des modalités qui varient en fonction des liens que chacun entretient avec le patrimoine qui la structure. Mais il reste que les sociétés rancheras n'ont jamais été entièrement repliées sur

14. Ley de la propiedad intelectual, chap. 2 de las marcas colectivas. Sistema de Informacion Sobre Comercio Exterior.www.sice.oas.org/int_prop/nat_leg/Mexico/

15. Information directe. Le processus engagé n'a pas encore abouti suite, notamment, à un veto opposé par le gouvernement de l'État de Michoacan. 
elles-mêmes. Leur ouverture sur l'extérieur qui a instruit la notoriété du fromage et, indirectement, induit la multiplication des contrefaçons peut églement, et à juste titre, en être considérée comme une caractéristique fondamentale [Barragan, 1987, Linck, 2001]. Le développement des échanges et l'unifiction des marchés mettent à jour une exigence de protection que les dispositifs de qualification sous IG promettent - en théorie - d'assurer. Mais, dans la logiques des normes internationales mises en place sous l'égide de l'OMC et des orientations libérales du gouvernement mexicain, cette protection reste plus qu'illusoire : elle est entièrement inscrite dans un double processus de marchandisation et de déconstruction patrimoniale [Barthélémy, 2004]. Qu'il s'agisse de marque collective ou de denominacion de origen, les dispositifs proposés nourrissent en effet une double logique de dissociation et de privatisation. La première tend à détacher le produit des activités d'amont, à le couper de son environnement naturel et social immédiat ainsi que de tout attribut non marchand : le queso Cotija, qui était originellement un fromage pastoral tend désormais à être défini comme une pâte pressée similaire au Cantal, qui n'a d'autre'valeur' que celle que le marché, à tort ou à raison, est disposé à reconnaître. La seconde peut être posée en corollaire : redéfini et dépouillé de ses attributs de bien patrimonial, le produit doit être réapproprié selon des modalité que dicte la confrontation des intérêts en présence.

Dans cette perspective, le parcours du queso Cotija révèle l'importance de la question de l'appropriation, de son sens et des dispositifs qui l'instruisent. Une première évidence s'impose : il n'y a pas d'appropriation sans exclusion. Les éleveurs des la Sierra de Jalmich l'ont bien compris : le règlement technique qu'ils ont conçu vise tout autant à valoriser une activité traditionnelle fortement ancrée dans le territoire qu'à exclure les élevages intensifs et les transformations non fermières. La position défendue par l'IMPI et les choix du gouvernement mexicains sont symétriques et tout aussi excluants : la pasteurisation, la non reconnaissance des productions fermières, le principe de généricité du fromage de Cotija. .. ne visent qu'à favoriser l'élevage intensif et la transformation industrielle aux dépens des productions familiales et artisanales. Les enjeux de l'exclusion sont tout aussi évidents. Il s'agit rien moins que de l'appropriation de ressources collectives : les savoirs et les pratiques pastoraux dans la mise en œuvre d'un projet de développement territorial d'un côté, l'exclusivité d'usage d'une dénomination et du bénéfice de la notoriété qui lui est accolée, de l'autre, dans une logique d'expansion industrielle. Considérée avec davantage de recul, l'exclusion peut alors s'inscrire dans les procès de l'accumulation primitive jadis portés par le mouvement des enclosures [Gorz, 1973 ; Azam, sf]

L'examen des dispositifs d'exclusion permet d'en souligner le caractère contingent. Telle qu'elle a pu être observée, l'exclusion relève du double registre des choix techniques et des options institutionnelles. Le premier prend un relief particulier dans un pays qui depuis près de soixante-dix ans a cessé de mettre en doute la neutralité du changement technique et son caractère nécessaire et linéaire [Linck, 1997]. La pasteurisation du lait, l'intensification et la standardisation des élevages, l'hygiène constituent pour les fonctionnaires et la plupart des 
universitaires des évidences sur lesquelles il est inutile de s'attarder. C'est pourtant bien au nom de ces principes que le gouvernement de l'État du Jalisco a lancé son projet de laiterie à contresens du règlement technique et des intérêts des éleveurs. Et c'est à ces mêmes éleveurs qu'il revient d'avoir montré que le sort du queso Cotija est intimement lié à l'autonomie et à la pugnacité du collectif en matière de choix techniques.

Le sort des démarches de qualification sous IG et leur impact potentiel en termes de développement territorial ne relèvent donc pas d'une simple question institutionnelle. Les institutions jouent pourtant un rôle important, pour une large part lié aux choix de société qui ont orienté leur construction. Ainsi, après avoir durant quelques années (jusqu'en 2004 approximativement) instruit un nombre élevé de dossiers de dénomination, l'IMPI semble s'être replié sur la conception ultra libérale qui tend à assimiler, dans son principe même, l'indication géographique à une distorsion de concurrence. Les dossiers en instruction ont été bloqués ou, comme le queso Cotija, rapidement réglé par l'octroi d'une marque collective qui, comme nous l'avons vu, n'offre qu'une protection très illusoire. Selon des responsables de l'IMPI, les « produits traditionnels tels que le queso Cotija, les guitares de Parcho, le cuivre de Santa Clara portent des'noms génériques' qui doivent rester libres et pouvoir être utilisés par n'importe quel individu ou entreprise ». Dans cette logique qui circonscrit strictement le champ de mise en œuvre des indications géographiques, les marques collectives elles-mêmes ne devraient pas mentionner la provenance du produit: l'origine, les savoirs locaux, les traditions... ne sauraient ainsi, en aucune façon, fonder une exclusivité d'usage ni par conséquent justifier une protection ou faire l'objet du moindre contrôle [Barragan, 2008] ${ }^{16}$.

Au-delà des choix en matière de protection de l'origine, le cadre institutionnel doit être considéré dans sa globalité. Le parcours du queso Cotija montre ainsi que les démarches engagées pour bénéficier d'un signe de qualité ont pour conséquence de « révéler » l'existence du produit et d'entraîner une obligation de respect des normes sanitaires, mais aussi comptables, fiscales et juridiques. Par là, le'système produit' - les savoirs techniques et organisationnels locaux, les activités liées, les circuits traditionnels de commercialisation - est extrait de la sphère des activités informelles pour être projeté dans le secteur de l'économie formelle. Il s'agit là d'une véritable mutation qui ne peut pas être engagée sans risque d'exclusion. D'une part parce que les acteurs concernés y sont d'autant moins préparés que l'ampleur de l'effort d'adaptation peut difficilement être anticipé. D'autre part, parce que le changement est entièrement inscrit dans un processus de marchandisation et donc marqué par le double sceau de la dissociation et de l'individualisation.

À l'échelle d'une procédure de qualification, les institutions ne se discutent pas, pas davantage que ne font débat les recettes productivistes qui sous-tendent

16. Entretien avec J. Khwagi (Responsable de la division «marques collectives » de l'IMPI) et A. Monjarras (Sous-directeur du département juridique et des indications géographiques de l'IMPI). 
les politiques publiques. Pourtant, les normes institutionnelles et techniques dominantes ne sont pas neutres, elles ont une incidence directe sur la définition du produit ainsi que sur la construction des modalités d'appropriation et de renouvellement des ressources. Le cadrage au final assez étroit du débat, les jeux d'exclusion et la divergence des intérêts en présence ne permettent pas de situer l'instruction d'un processus de qualification dans le registre de la concertation. Loin de l'idéal parétien qui veut que tous les espoirs de changement sont permis du moment que personne ne subit de perte nette, le processus est porté par des logiques d'accaparement et d'éviction : il s'inscrit donc bien davantage dans une logique de conflit. Ce conflit qui a pour enjeu l'appropriation et l'entretien des patrimoines territoriaux est à inscrire dans le registre des luttes agraires. Tel est bien l'enseignement qui peut être retiré du parcours du queso Cotija. Le combat des éleveurs de la Sierra de Jalmich pour la reconnaissance de leur produit est aussi, au plein sens du terme, un combat pour la terre.

\section{BibLIOGRAPHIE}

Azam G., s.f. «Les droits de propriété sur le vivant » Département d'économie, Université de Toulouse le Mirail, doc ronéoté.

Barragan E. [1990], Más allá de los caminos, El Colegio de Michoacán, Zamora.

BARRAGAn E. [1997], Con un pie en el estribo. Formación y deslizamientos de las sociedades rancheras en la construcción del México moderno, Zamora, El Colegio de Michoacán.

BARRAGAN E. [2008], «El IMPI no se decide a proteger los productos tradicionales en México » Guia, Zamora, 15 juin 2008.

BARRAGAN E., LinCK T. [1993], «Quinientos años de soledad II. Sociedad y poblamiento ranchero » Trace CEMCA, México.

Barragan E., Chavez M. [2005], «El queso Cotija se nos va de las manos »El Colegio de Michoacán, Zamora.

Barragan E., Chavez M., Linck T. [à paraître], « Le choix technique comme enjeu de patrimonialisation : l'exemple du fromage de Cotija » PIDAL, INRA, Paris.

Barthélémy D., Nieddu M., Vivien F.-D. [2004], «Économie patrimoniale, identité et marché », in Repenser le patrimoine, L'Harmattan, Paris.

Boucher F. [2008], «La leche como instrumento de desarrollo territorial de los pequenos productores para acceder a nuevos mercados » in Castelan Ortegan (ed.) Oportunidades y retos para los sistemas campesinos de rumiantes en Latino-américa. Manejo de recursos, seguridad alimentaria, calidad y acceso a mercados urbanos, UAEM, Toluca (Mexique).

Chevalier F. [2006], La formation des grands domaines au Mexique. Terre et société, XVI ${ }^{-}$XVII'-XVIII siècles, Paris, Khartala.

Cochet H. [1991], Alambradas en la sierra., CEMCA-ORSTOM-COLMICH, Mexico.

George P. [1963], Précis de géographie rurale, Paris, PUF.

Gonzalez Mendez V., Ortiz Ybarra H. [1980], Los Reyes, Tingüindin, Tancitaro, Tocumbo y Periban. Monografias municipales, Gobierno del Estado de Michoacan, Morelia.

Gorz A. [1973], Critique de la division du travail, Seuil, Paris. 
FISChLER C. [2001], L'homnivore, Odile Jacob, Paris.

KATZ, F. [1991], El mundo rural mexicano a través de los siglos., Universidad de Guadalajara, Guadalajara.

LincK T. [1988], El campesino desposeido, El Colegio de Michoacán - CEMCA, Mexico.

LINCK T. [2001], « Conquête et colonisation des montagnes sèches mexicaines. La production des territoires et des sociétés rancheras ", Les montagnes tropicales. Identités, mutations, développement, Espaces tropicaux, $\mathrm{n}^{\circ}$ 16, Bordeaux, p. 631-640.

LiNCK T. [1997], «La ruralité en miettes. Globalisation et recompositions territoriales au Mexique », in J.-M. Gastellu et J.-Y. Marchal, La ruralité dans les pays du sud à la fin $d u$ $X X^{e}$ siècle, ORSTOM, Paris.

LINCK T. [2005], «Patrimonialisation et typification de fromages «traditionnels » : une approche comparée de démarches de qualification », Ruralia, 2005-16/17.

LiNCK T. [2007], « Patrimoines sous tension. L'exclusion, condition et écueil de l'appropriation collective », Économie appliquée, Paris.

Linck T., Barragan E. [2008], «Le queso Cotija, le fromage de la conquête des Sierras de l'ouest mexicain », Fora, Ajaccio.

LLOYD J. D. [1988] «Desarrollo histórico del ranchero » $y$ «Rancheros y revolucionarios en Chihuahua ». Historia de la cuestión agraria mexicana, campesinos, terratenientes y revolucionarios 1810-1910, T. 3, Siglo XXI-CEHAM, Mexico.

Marie-VIVIen D., ThÉVENod-Mottet E. [2007], Une décision de l'organe de règlement des différends de l'OMC. Quels impacts pour la protection internationale des indications géographiques ?, "Enjeux internationaux et institutionnels des signes de qualité et d'origine », Economie rurale, Agriculture, Alimentations, Territoires, $\mathrm{n}^{\circ}$ 299, mai-juin 2007, p. 58-69.

Moreno Garcia H. [1980], Cotija, Monografías municipales. Gobierno del Estado de Michoacán.

Pomeon T. [2007], El Queso Cotija México. Un producto con marca colectiva queso « Cotija, region de origen » en proceso de adquisicion de una denominacion de origen. CIESTAM Universidad Autonoma Chapingo. FAO, IICA, Mexico.

Poulain J.-P. [2005], Sociologies de l'alimentation : les mangeurs et l'espace social alimentaire. Privat.

RODRIGUEZ Gómez G. [2002], La denominación de origen y el mercado de la distinción, CIESAS/SAGARPA, México.

SHADOw R. D. [1990], «Los Rancheros del Occidente : hacia un modelo de su organización comunitaria », Primer Coloquio de Occidentalistas, Universidad de Guadalajara (mimeo) Guadalajara. 